\title{
Influence of the forms and levels of dietary selenium on antioxidant status and oxidative stress-related parameters in rainbow trout (Oncorhynchus mykiss) fry
}

\author{
Stéphanie Fontagné-Dicharry ${ }^{1 *}$, Simon Godin ${ }^{2}$, Haokun Liu ${ }^{1,3}$, Philip Antony Jesu Prabhu ${ }^{1}$, \\ Brice Bouyssière ${ }^{2}$, Maïté Bueno ${ }^{2}$, Philippe Tacon ${ }^{4}$, Françoise Médale ${ }^{1}$ and Sadasivam J. Kaushik ${ }^{1}$ \\ ${ }^{1}$ INRA, UR1067 NuMéA, F-64310 St-Pée-sur-Nivelle, France \\ ${ }^{2}$ LCABIE UMR5254, F-64053 Pau Cedex 09, France \\ ${ }^{3}$ FPE, Institute of Hydrobiology, CAS, 430-072 Wuban, People's Republic of China \\ ${ }^{4}$ Lesaffre Feed Additives, F-59700 Marcq-en-Baroul, France
}

(Submitted 8 December 2014 - Final revision received 23 March 2015 - Accepted 26 March 2015 - First published online 20 May 2015)

\section{Abstract}

Se is an essential micronutrient required for normal growth, development and antioxidant defence. The objective of the present study was to assess the impact of dietary Se sources and levels on the antioxidant status of rainbow trout (Oncorbynchus mykiss) fry. First-feeding fry (initial body weight: $91 \mathrm{mg}$ ) were fed either a plant- or fishmeal-based diet containing 0.5 or $1.2 \mathrm{mg}$ Se/kg diet supplemented or not with $0.3 \mathrm{mg} \mathrm{Se} / \mathrm{kg}$ diet supplied as Se-enriched yeast or sodium selenite for 12 weeks at $17^{\circ} \mathrm{C}$. Growth and survival of rainbow trout fry were not significantly affected by dietary Se sources and levels. Whole-body Se was raised by both Se sources and to a greater extent by Se-yeast. The reduced:oxidised glutathione ratio was raised by Se-yeast, whereas other lipid peroxidation markers were not affected by dietary Se. Whole-body Se-dependent glutathione peroxidase (GPX) activity was enhanced in fish fed Se-yeast compared to fish fed sodium selenite or non-supplemented diets. Activity and gene expression of this enzyme as well as gene expression of selenoprotein P (SelP) were reduced in fish fed the non-supplemented plant-based diet. Catalase, glutamate-cysteine ligase and nuclear factor-erythroid 2-related factor 2 (Nrf2) gene expressions were reduced by Se-yeast. These results suggest the necessity to supplement plant-based diets with Se for rainbow trout fry, and highlight the superiority of organic form of Se to fulfil the dietary Se requirement and sustain the antioxidant status of fish. GPX and SelP expression proved to be good markers of Se status in fish.

Key words: Selenium: Oxidative stress: Glutathione peroxidase: Rainbow trout fry

Se is an essential micronutrient for animals and humans required for normal growth and development ${ }^{(1,2)}$. This role is attributed to low molecular weight Se compounds, as well as to the presence of Se within proteins and enzymes, named selenoproteins, in the form of the amino acid selenocysteine. Mammals have at least twenty-five selenoproteins, and fish probably more than thirty-two, although the functions of many selenoproteins are not yet elucidated ${ }^{(3,4)}$. Selenoproteins are involved in diverse physiological functions such as antioxidant defence, reduction of inflammation, thyroid hormone production, DNA synthesis, fertility and reproduction ${ }^{(1)}$. As a component of glutathione peroxidases (GPX), thioredoxin reductases (TR), and methionine sulphoxide reductases (MSR), Se plays, in particular, a pivotal role against oxidative cellular injury and lipid peroxidation. GPX can reduce $\mathrm{H}_{2} \mathrm{O}_{2}$ and organic hydroperoxides to the corresponding alcohols with oxidation of glutathione to glutathione disulphide ${ }^{(5)}$. Seven GPX have been described in mammals, five of which are selenoproteins ${ }^{(2)}$. The GPX selenoproteins include the ubiquitously expressed cytosolic GPX1, a gastrointestinal-specific enzyme GPX2, a secreted protein found in plasma GPX3, a ubiquitously expressed enzyme that acts on oxidised lipids named

\footnotetext{
Abbreviations: CAT, catalase; F, fishmeal-based diets; FSe0, fishmeal-based diet not supplemented with Se; FSeS, fishmeal-based diet supplemented with sodium selenite; FSeY, fishmeal-based diet supplemented with Se-enriched yeast; GPX, glutathione peroxidase; GSH, reduced glutathione; GSSG, oxidised glutathione; GST, glutathione-S-transferase; MSR, methionine sulphoxide reductase; Nrf2, nuclear factor erythroid-2-related factor 2; P, plantbased diets; PSe0, plant-based diet not supplemented with Se; PSeS, plant-based diet supplemented with sodium selenite; PSeY, plant-based diet supplemented with Se-enriched yeast; Se-GPX, Se-dependent glutathione peroxidase; SelP, selenoprotein P; SOD, superoxide dismutase; TR, thioredoxin reductase.
}

* Corresponding author: S. Fontagné-Dicharry, fax + 33 559545152, email fontagne@st-pee.inra.fr 
phospholipid hydroperoxide GPX or GPX4, and the GPX6 located in olfactory epithelium and embryonic tissues. GPX1 and GPX4 genes have been cloned in rainbow trout revealing several isoforms ${ }^{(6)}$. TR can act by controlling the function of thioredoxin, a central redox molecule, and by directly reducing numerous substrates including lipid hydroperoxides ${ }^{(2)}$. Three Se-containing TR have been identified in mammals with TR1, the most abundant isoenzyme found in the cytosol, TR2, a mitochondrial form and TR3 found predominantly in the testis ${ }^{(7)}$. MSR repair oxidatively damaged proteins and protect against oxidative stress ${ }^{(8)}$. MSR include two stereospecific enzyme families: MSRA specific for reduction of the $S$-epimer of methionine sulphoxide, and MSRB which can only act on the $R$-form. Three selenoprotein MSRB genes have been identified in mammals with MSRB1 also known as selenoprotein $\mathrm{R}$ or $\mathrm{X}$ present in the cytosol and nucleus, MSRB2 targeted to the mitochondria and MSRB3 localised in the endoplasmic reticulum. In addition, selenoprotein $\mathrm{P}$ (SelP), present in the plasma of mammals and expressed in cellular membranes, is regarded as a transport protein for Se but also has antioxidant properties, as it can directly reduce phospholipid hydroperoxides in vitro ${ }^{(9,10)}$.

Dietary Se deficiency can influence the level of activity of several selenoproteins, and in some cases also cause changes in mRNA levels for selenoprotein genes ${ }^{(3,11,12)}$. If Se intake is suboptimal, selenoprotein functions may be impaired, resulting in altered Se metabolism. In fish, a dietary Se deficiency has generally been reported to result in reduced activity of GPX, as well as growth reduction ${ }^{(13)}$ and increased mortality ${ }^{(14)}$. Muscular dystrophy and exudative diathesis have also been observed in channel catfish, when dietary Se deficiency was combined with dietary vitamin E deficiency ${ }^{(15)}$. On the other hand, Se may exert toxic effects at levels marginally above those required ${ }^{(13,16)}$. A dietary Se requirement varying from 0.15 to $0.7 \mathrm{mg} / \mathrm{kg}$ diet has been reported for different fish species based on weight gain and GPX activity with threshold levels for adverse effects estimated at $3-4 \mathrm{mg} \mathrm{Se} / \mathrm{kg}$ diet $^{(13)}$. This dietary Se requirement might be higher in early life stages of fish exhibiting a high growth rate as suggested by Bell et al. ${ }^{(17)}$ to explain the absence of pathology in rainbow trout juveniles, compared to Atlantic salmon fry fed low dietary Se levels. The chemical form of Se is known to strongly affect its bioavailability and its impact on metabolism ${ }^{(6,18,19)}$. Animal feeds can be supplemented with inorganic (e.g. selenite, selenate) or organic (e.g. selenomethionine, selenocysteine) Se to a maximum authorised level of $0.3 \mathrm{mg} / \mathrm{kg}$ according to US Food and Drug Administration ${ }^{(20)}$, and $0 \cdot 2 \mathrm{mg} / \mathrm{kg}$ according to European Food Safety Authority ${ }^{(21)}$. Fish meal-based diets generally provide sufficient Se to satisfy the nutritional requirements of fish ${ }^{(16)}$, but can contain levels of Se well above the maximum dietary levels permitted by European Food Safety Authority $(0.5 \mathrm{mg} / \mathrm{kg})$. However, currently, there is significant reduction in fishmeal levels in fish feeds, particularly in salmonid feeds, replaced to a large extent by plant ingredients $^{(22)}$ that vary widely in their Se content ${ }^{(16,23)}$.

The objective of the study was hence to assess the impact of dietary Se supplementation by an organic or inorganic source on the antioxidant status of rainbow trout (Oncorbynchus mykiss) fry fed practical plant protein or fishmeal-based feeds.

\section{Materials and methods}

\section{Diets}

Ingredients were mixed and the diets were manufactured using a twin-screw extruder (BC 45, Clextral) at the INRA experimental facilities in Donzacq (Landes, France). Six diets were formulated with two different basal ingredient compositions (Table 1). P-diets were based on plant-derived proteins and vegetable oils, and F-diets were formulated to contain fishmeal and fish oil as protein and lipid sources. The P-diets were supplemented with $1 \%$ mineral mixture to meet all the essential mineral requirements of rainbow trout $^{(13)}$, except for Se. F-diets were not supplemented with mineral mixture, as fishmeal represents a major source of minerals and trace elements; high $\mathrm{Ca}$ or $\mathrm{P}$ levels have been shown to adversely affect absorption of some trace elements in different fish species ${ }^{(13)}$; high levels of other trace elements such as $\mathrm{Cu}$ and $\mathrm{Zn}$ have been shown as well to interfere with $\mathrm{Se}^{(24-26)}$. SeS- and SeY-diets were supplemented with either sodium selenite (SeS-diets) or the Se-enriched yeast Selsaf (SeY-diets) at a concentration of $0.3 \mathrm{mg} \mathrm{Se} / \mathrm{kg}$ feed. PSe0- and FSe0-diets containing 0.5 and $1.2 \mathrm{mg} \mathrm{Se} / \mathrm{kg}$ feed respectively, were not supplemented with Se and served as controls.

\section{Fish}

Eyed-embryos of all-female diploid rainbow trout (O. mykiss) were provided by Viviers de Sarrance to the INRA experimental fish farm in Donzacq (Landes, France). Rainbow trout fry were randomly allocated to eighteen fibreglass tanks (50 litres) supplied with flow-through spring water at $17^{\circ} \mathrm{C}$ with 200 fish/tank. From the swim-up stage, which corresponds to the beginning of exogenous feeding, fish (initial body weight: $91 \mathrm{mg}$ ) were hand-fed six times a day to apparent satiation. Each diet was distributed to three replicate groups of fish over a 12-week growth trial. For sampling at the start and the end of the feeding trial, fish were feed-deprived for $16 \mathrm{~h}$, killed with an overdose of benzocaine, weighed, frozen in liquid $\mathrm{N}_{2}$ and stored at $-80^{\circ} \mathrm{C}$ before analysis. All experimental procedures complied with the European Directive 010/63/EU for the protection of animals used for scientific purposes, and the French Decree no. 2001-464 for animal experimentation.

\section{Determination of proximate, mineral and fatty acid composition}

Proximate composition of diets and whole fish was determined according to the following procedures: DM after drying at $105^{\circ} \mathrm{C}$ for $24 \mathrm{~h}$, protein $(\mathrm{N} \times 6.25)$ by the Kjeldahl method after acid digestion ${ }^{(27)}$, ash by incineration at $550^{\circ} \mathrm{C}$ for $10 \mathrm{~h}$ and gross energy in an adiabatic bomb calorimeter. Total lipid was extracted and measured gravimetrically according to Folch et al. ${ }^{(28)}$ using dichloromethane instead 
Table 1. Formulation and composition of the experimental diets $(\mathrm{g} / 100 \mathrm{~g}$ dry weight)

\begin{tabular}{|c|c|c|c|c|c|c|}
\hline Diet & PSe0 & PSeS & PseY & FSe0 & FSeS & $\mathrm{FSeY}$ \\
\hline \multicolumn{7}{|l|}{ Ingredients } \\
\hline Plant meals and crystalline amino acids* & $74 \cdot 8$ & 74.8 & 74.8 & - & - & - \\
\hline Fish meal $\dagger$ & - & - & - & $62 \cdot 6$ & $62 \cdot 6$ & $62 \cdot 6$ \\
\hline Whole wheat $\ddagger$ & $2 \cdot 0$ & $2 \cdot 0$ & $2 \cdot 0$ & 24.5 & 24.5 & 24.5 \\
\hline Vegetable oils§ & 10.5 & $10 \cdot 5$ & 10.5 & - & - & - \\
\hline Fish oilt & $5 \cdot 0$ & $5 \cdot 0$ & $5 \cdot 0$ & $11 \cdot 9$ & 11.9 & 11.9 \\
\hline Dicalcium phosphate & $2 \cdot 2$ & $2 \cdot 2$ & $2 \cdot 2$ & - & - & - \\
\hline Soyabean lecithin\| & $2 \cdot 0$ & $2 \cdot 0$ & $2 \cdot 0$ & - & - & - \\
\hline Attractant mixtureq & 1.5 & 1.5 & 1.5 & - & - & - \\
\hline Vitamin mixture ${ }^{\star \star}$ & 1.0 & 1.0 & $1 \cdot 0$ & 1.0 & 1.0 & 1.0 \\
\hline Mineral mixture without Se†† & 1.0 & 1.0 & 1.0 & - & - & - \\
\hline Sodium selenite ( $\mu \mathrm{g} / \mathrm{g}$ diet) & - & 0.7 & - & - & 0.7 & - \\
\hline Se-enriched yeast $\neq \ddagger(\mu \mathrm{g} / \mathrm{g}$ diet) & - & - & 150 & - & - & 150 \\
\hline \multicolumn{7}{|l|}{ Analytical composition } \\
\hline DM (\%) & $92 \cdot 2$ & 91.9 & $92 \cdot 3$ & $92 \cdot 1$ & $92 \cdot 0$ & 91.9 \\
\hline Crude protein & $48 \cdot 5$ & 47.5 & $48 \cdot 5$ & $47 \cdot 0$ & $47 \cdot 3$ & $47 \cdot 2$ \\
\hline Total lipid & $22 \cdot 8$ & $23 \cdot 0$ & 23.5 & $21 \cdot 7$ & $21 \cdot 8$ & 21.7 \\
\hline Gross energy (kJ/g DM) & $24 \cdot 3$ & $24 \cdot 7$ & 24.9 & $22 \cdot 9$ & 23.4 & $23 \cdot 6$ \\
\hline Ash & $5 \cdot 0$ & $5 \cdot 2$ & $5 \cdot 2$ & 11.4 & 11.5 & $9 \cdot 1$ \\
\hline Total P & 0.9 & 0.9 & $1 \cdot 0$ & 1.7 & 1.7 & 1.4 \\
\hline Total Se ( $\mu \mathrm{g} / \mathrm{g}$ diet $)$ & 0.5 & 0.9 & 0.9 & $1 \cdot 2$ & 1.6 & $1 \cdot 7$ \\
\hline
\end{tabular}

PSe0, plant-based diet not supplemented with Se; PSeS, plant-based diet supplemented with sodium selenite; PSeY, plant-based diet supplemented with Se-enriched yeast; FSe0, fishmeal-based diet not supplemented with Se; FSeS, fishmeal-based diet supplemented with sodium selenite; FSeY, fishmeal-based diet supplemented with Se-enriched yeast.

* Plant meals (\% diet): $20 \%$ wheat gluten (Roquette); $18 \%$ corn gluten meal (Inzo); $15 \%$ soyabean protein concentrate Estrilvo 70 (Sopropêche); $6.2 \%$ soyabean meal (Sud-Ouest Aliment); $5.2 \%$ rapeseed meal 00 (Sud-Ouest Aliment); $5 \%$ white lupin meal (Terrena); $3.8 \%$ dehulled pea meal Primatex (Sotexpro); $1.3 \%$ L-lysine (Ajinomoto-Eurolysine); $0.3 \%$ L-methionine (Evonik).

† Norwegian herring meal Norse LT94 and capelin oil from southern hemisphere supplied by Sopropêche.

$¥$ Supplied by Sud-Ouest Aliment.

$\S$ Vegetable oils (\% diet): $4 \cdot 2 \%$ rapeseed oil; $4 \cdot 2 \%$ linseed oil; $2 \cdot 1 \%$ palm oil (Daudry).

\| Supplied by Louis François.

I Attractant mixture (g/kg diet): glucosamine, 5; taurine, 3; betaine, 3; glycine, 2; alanine, 2.

${ }^{\star *}$ Vitamin mixture (per $\mathrm{kg}$ diet): retinol acetate, $1.5 \mathrm{mg}$; cholecalciferol, $62.5 \mu \mathrm{g}$; DL- $\alpha$-tocopherol acetate, $50 \mathrm{mg}$; sodium menadione bisulphate, $10 \mathrm{mg}$; thiamine- $\mathrm{HCl}, 1 \mathrm{mg}$; riboflavin, $4 \mathrm{mg}$; niacin, $10 \mathrm{mg}$; D-calcium panthothenate, $20 \mathrm{mg} ; \mathrm{pyridoxine-HCl,} 3 \mathrm{mg}$; meso-inositol, $300 \mathrm{mg}$; D-biotin, $0.2 \mathrm{mg}$; folic acid, $1 \mathrm{mg}$; cyanocobalamin, $10 \mu \mathrm{g}$; L-ascorbyl-2-polyphosphate $50 \mathrm{mg}$; choline, $1 \mathrm{~g}$. All ingredients were diluted with $\alpha$-cellulose.

†† Mineral mixture (per kg diet): $\mathrm{CaHPO}_{4} \cdot 2 \mathrm{H}_{2} \mathrm{O}, 5 \mathrm{~g} ; \mathrm{CaCO}_{3}, 2.15 \mathrm{~g} ; \mathrm{NaCl}, 0.4 \mathrm{~g} ; \mathrm{FeSO}_{4} .7 \mathrm{H}_{2} \mathrm{O}, 0.2 \mathrm{~g} ; \mathrm{MnSO}_{4} \cdot \mathrm{H}_{2} \mathrm{O}, 30 \mathrm{mg} ; \mathrm{ZnSO}_{4} .7 \mathrm{H}_{2} \mathrm{O}$, $40 \mathrm{mg} ; \mathrm{CuSO}_{4} .5 \mathrm{H}_{2} \mathrm{O}, 30 \mathrm{mg} ; \mathrm{CoCl}_{2} .6 \mathrm{H}_{2} \mathrm{O}, 0.2 \mathrm{mg} ; \mathrm{KI}, 0.4 \mathrm{mg} ; \mathrm{NaF}, 10 \mathrm{mg} ; \mathrm{MgOH}, 1.24 \mathrm{~g} ; \mathrm{KCl}, 0.9 \mathrm{~g}$. All ingredients were diluted with $\alpha$-cellulose.

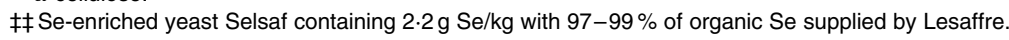

of chloroform. Total P was determined by the molybdateblue/ ascorbic acid method at $820 \mathrm{~nm}$ after mineralisation and acid digestion $^{(29)}$. For total Se determination, diet and whole fry samples were first subjected to acid digestion using concentrated $\mathrm{HNO}_{3}$ and $\mathrm{H}_{2} \mathrm{O}_{2}$, samples were then diluted with water until a concentration of $2 \% \mathrm{HNO}_{3}$ was reached, and total Se concentration was measured in the digesta by inductively coupled plasma MS (Agilent 7500ce; Yokogawa Analytical Systems) ${ }^{(30)}$. The instrument was equipped with a collision/reaction cell, and $\mathrm{H}_{2}$ was used as reaction gas in order to remove interferences from argon dimers. Matrix effects were corrected by the use of internal calibration, and Ga was used as internal standard. Fatty acid methyl esters were prepared and analysed as described previously ${ }^{(31)}$. The concentration of the individual fatty acid was expressed in percentage of total fatty acids.

\section{Determination of oxidative status}

Peroxide value of total lipid from diets or whole fish was assessed by colorimetric determination of iron-thiocyanate according to Shantha \& Decker ${ }^{(32)}$. Conjugated dienes $\left(\mathrm{E}_{232}\right)$ and trienes $\left(E_{268}\right)$ were measured as specific extinctions at the wavelengths of 232 and $268 \mathrm{~nm}$, respectively ${ }^{(33)}$. Anisidine value was determined according to standard procedures ${ }^{(34)}$. For measurement of lipid-soluble fluorescent products, fluorescence intensity of $10 \mathrm{mg}$ total lipid diluted in $1 \mathrm{ml}$ chloroform-methanol (7:3, v/v) was determined in a spectrofluorometer (Triad Dynex, Serlabo), using excitation/emission wavelengths of $360 / 465 \mathrm{~nm}$, and quinine sulphate as standard at a concentration of $1 \mu \mathrm{g} / \mathrm{ml}$ in $0 \cdot 05 \mathrm{~m}$-sulphuric acid ${ }^{(35,36)}$. Oxidised glutathione (GSSG) and reduced glutathione (GSH) were measured in whole fish homogenates using Cayman glutathione assay kit (Bertin Pharma) according to the manufacturer's instructions.

\section{Determination of antioxidant enzyme activity}

Antioxidant enzyme activities were assayed in whole fish as described previously ${ }^{(37)}$. Briefly, superoxide dismutase (SOD, EC 1.15.1.1) activity was measured by monitoring the inhibition of nitrotetrazolium reduction at $550 \mathrm{~nm}$, and was expressed as the amount of enzyme required to inhibit the rate of nitrotetrazolium reduction by $50 \% / \mathrm{mg}$ protein at $37^{\circ} \mathrm{C}$. Catalase (CAT, EC 1.11.1.6) activity was measured by monitoring the decomposition of $\mathrm{H}_{2} \mathrm{O}_{2}$ at $240 \mathrm{~nm}$, and was expressed as $\mu \mathrm{mol} \mathrm{H}_{2} \mathrm{O}_{2}$ reduced $/ \mathrm{min}$ per $\mathrm{mg}$ protein at $30^{\circ} \mathrm{C}$. GPX (EC 1.11.1.9) activity 
was assayed by the coupled reaction with glutathione reductase (GR) using cumene hydroperoxide and $\mathrm{H}_{2} \mathrm{O}_{2}$ as substrates for measuring total GPX and Se-dependent GPX (Se-GPX) respectively, and was expressed as nmol NADPH oxidised/min per $\mathrm{mg}$ protein at $30^{\circ} \mathrm{C}$. GR (EC 1.6.4.2) activity was determined by monitoring NADPH oxidation at $340 \mathrm{~nm}$, and was expressed as nmol NADPH oxidised/min per $\mathrm{mg}$ protein at $30^{\circ} \mathrm{C}$. Glutathione-S-transferase (GST, EC 2.5.1.18) activity was assayed by following the conjugation of glutathione with 1-chloro-2,4-dinitrobenzene at $340 \mathrm{~nm}$, and was expressed as nmol $S$-2,4-dinitrophenylglutathione formed/min per $\mathrm{mg}$ protein at $30^{\circ} \mathrm{C}$. TR (EC 1.8.1.9) activity was determined by monitoring the reduction of 2-nitrobenzoic acid with NADPH to 5-thio-2-nitrobenzoic acid at $412 \mathrm{~nm}$, and was expressed as increase in absorbance at $412 \mathrm{~nm} / \mathrm{min}$ per $\mathrm{mg}$ protein at $\mathrm{pH} 7.0$ at $25^{\circ} \mathrm{C}$. Protein concentration was determined by the method of Lowry et al. ${ }^{(38)}$ using bovine serum albumin as a standard.

\section{Determination of antioxidant enzyme expression}

Total RNA was isolated from whole fish using Trizol reagent (Invitrogen). For quantitative RT-PCR, complementary DNA was generated from $1 \mu \mathrm{g}$ total RNA using SuperScript ${ }^{\circledR}$ III RT (Invitrogen) and a mix of oligo(dT) 15 and random primers (Promega). For each sample, RT was performed in duplicate and quantitative PCR analyses were performed in duplicate using the iQTM SYBR ${ }^{\circledR}$ Green Supermix (Bio-Rad) in a total volume of $15 \mu \mathrm{l}$ containing $5 \mu \mathrm{l}$ of the diluted RT reaction mixture (dilution 64) and $200 \mathrm{~nm}$ of each primer (Table 2) as described previously ${ }^{(39)}$.

\section{Statistical analyses}

Results are shown as the means with their standard errors. Differences between dietary groups were evaluated using

Table 2. Oligonucleotide primers used to assay gene expression by quantitative RT-PCR two-way ANOVA followed by Student-Newman-Keuls post boc analysis to test the effects of Se supplementation and dietary basis. As interaction between Se supplementation and dietary bases ( $\mathrm{P}$ or $\mathrm{F}$ ) was found to be significant for some parameters, differences between dietary groups are shown using one-way ANOVA and the main results of twoway ANOVA are presented in Table 3. Percentage data were arc-sin transformed, and weight data were log-transformed before analysis. For gene expression analysis, relative quantification of the target gene transcript with the $\beta$-actin reference gene transcript was performed using the $\Delta \Delta C_{\mathrm{t}}$ method $^{(40)}$. Statistical analyses were performed with the computing program Statbox (Grimmer Logiciels) and results with $P<0.05$ were considered significant.

\section{Results}

\section{Growth performance and whole-body composition}

Rainbow trout fry readily accepted the experimental diets from the beginning of the investigation, and maintained normal behaviour throughout the growth trial. As the fish were fed to excess or visual satiety, feed intake and feed efficiency were not calculated, but these parameters were evaluated to check that no group was underfed. The survival remained high $(>94 \%)$ by day 15 for all dietary treatments. From day 67 onwards, survival was significantly lower in fish fed F-diets than in fish fed P-diets (87v. 91\%), but was not significantly affected by dietary se sources and levels (Table 3). Mean wet weight increased approximately ninety-fold during the 12 -week feeding trial and no significant differences were recorded between dietary groups (Table 3).

Final whole-body composition was significantly affected by dietary Se supplementation and dietary basis according to two-way ANOVA with the exception of crude protein (Table 3). Whole-body lipid content increased from 4.2 to $10 \cdot 5 \%$

\begin{tabular}{|c|c|c|c|c|c|}
\hline \multirow[b]{2}{*}{ Gene } & \multicolumn{2}{|c|}{$5^{\prime}-3^{\prime}$ Primer sequence } & \multirow[b]{2}{*}{ Amplicon size (bp) } & \multirow[b]{2}{*}{ Annealing temperature $\left({ }^{\circ} \mathrm{C}\right)$} & \multirow[b]{2}{*}{ GenBank accession no. } \\
\hline & Forward & Reverse & & & \\
\hline$G P X 1 a^{*}$ & aatgtggcgtcactctgagg & caattctcctgatggccaaa & 131 & 56 & HE687021 \\
\hline$G P X 1 b 1^{*}$ & cgagctccatgaacggtacg & tgcttcccgttcacatccac & 183 & 59 & СА357669.1 \\
\hline$G P X 1 b 2^{*}$ & tcggacatcaggagaactgc & tccttcccattcacatccac & 121 & 56 & HE687023 \\
\hline GPX4a1* & gaaaggcttcctgggaaatg & ctccaccacactgggatcat & 112 & 56 & HE687024 \\
\hline$G P X 4 a 2^{*}$ & agaaatacaggggegacgtt & gcatctccgcaaactgagag & 90 & 56 & HE687025 \\
\hline$G P X 4 b^{\star}$ & ttggaggtcaggagccaggt & accctttcccttgggetgtt & 152 & 59 & CA344428.1 \\
\hline SelP & accttgctgagccagaaact & cagacgaccacacctgtcat & 129 & 55 & ВТ072678.1 \\
\hline MSRB2 & aggggacagagatgcccttc & cccatgagcctctttgaacg & 149 & 58 & CA354807.1 \\
\hline TR1 & gagcctccctcaagtgtgac & agtgaactccagaggccaga & 158 & 55 & СА342676.1 \\
\hline$S O D 1$ & tggtcctgtgaagctgattg & ttgtcagctcctgcagtcac & 201 & 56 & AF469663.1 \\
\hline SOD2 & tccctgacctgacctacgac & ggcctcctccattaaacctc & 201 & 56 & CA352127.1 \\
\hline$C A T$ & tgatgtcacacaggtgcgta & gtgggctcagtgttgttgag & 195 & 55 & BX087110.3 \\
\hline$G R$ & ctaagcgcagcgtcatagtg & acacccctgtctgacgacat & 108 & 55 & CA368976.1 \\
\hline GST & tcgctgactggacgaaagga & cgaaggtcctcaacgccatc & 196 & 59 & BX302932.3 \\
\hline Gclc & aggccagagtatggcagcta & ttgggaatgatgtgatggtg & 166 & 55 & GE829695.1 \\
\hline Nrf2 & tgagctgcagcaatgtctga & gttgggcaatgggtagaagc & 124 & 57 & HQ916348.1 \\
\hline$\beta$-Actin & gatgggccagaaagacagcta & tcgtcccagttggtgacgat & 105 & 59 & AJ438158.1 \\
\hline
\end{tabular}

GPX, glutathione peroxidase; SeIP, selenoprotein P; MSRB, methionine-R-sulphoxide reductase B; TR, thioredoxin reductase; SOD, superoxide dismutase; $C A T$, catalase; GR, glutathione reductase; GST, glutathione-S-transferase; Gclc, glutamate-cysteine ligase catalytic subunit; Nrf2, nuclear factor erythroid-2-related factor 2. ${ }^{*}$ As described by Pacitti et $a l^{(6)}$ 
Table 3. Growth performance, final whole-body composition, glutathione content, antioxidant enzyme activity and gene expression of rainbow trout fry fed the experimental diets for 12 weeks (Mean values with their standard errors; $n 6$ tanks per selenium supplementation and $n 9$ tanks per dietary basis)

\begin{tabular}{|c|c|c|c|c|c|c|c|c|c|c|c|}
\hline & \multicolumn{6}{|c|}{ Se supplementation } & \multicolumn{4}{|c|}{ Dietary basis } & \multirow{3}{*}{$\begin{array}{c}\text { Se supplementation } \times \text { dietan } \\
\text { basis interaction: } P\end{array}$} \\
\hline & \multicolumn{2}{|c|}{$\mathrm{Se} 0$} & \multicolumn{2}{|c|}{ Ses } & \multicolumn{2}{|c|}{$\mathrm{SeY}$} & \multicolumn{2}{|c|}{$P$} & \multicolumn{2}{|c|}{$\mathrm{F}$} & \\
\hline & Mean & SEM & Mean & SEM & Mean & SEM & Mean & SEM & Mean & SEM & \\
\hline \multicolumn{12}{|l|}{ Growth performance } \\
\hline Final survival (\%) & 87 & 2 & 87 & 2 & 83 & 1 & $91^{\mathrm{a}}$ & 1 & $80^{\mathrm{b}}$ & 1 & NS \\
\hline Final body weight (g) & 7.5 & 0.2 & $7 \cdot 3$ & 0.2 & $7 \cdot 2$ & 0.2 & $7 \cdot 3$ & 0.2 & $7 \cdot 3$ & 0.1 & NS \\
\hline \multicolumn{12}{|l|}{ Whole-body composition } \\
\hline DM (\%) & $25 \cdot 1^{\mathrm{b}}$ & 0.2 & $25 \cdot 9^{\mathrm{a}}$ & 0.1 & $26 \cdot 0^{\mathrm{a}}$ & 0.1 & $26 \cdot 1^{\mathrm{a}}$ & 0.2 & $25 \cdot 2^{b}$ & 0.1 & NS \\
\hline Total lipid (\%) & $10 \cdot 0^{\mathrm{b}}$ & $0 . \overline{2}$ & $10 \cdot 8^{a}$ & 0.1 & $10 \cdot 7^{\mathrm{a}}$ & 0.1 & $11 \cdot 2^{\mathrm{a}}$ & 0.1 & $9 \cdot 8^{b}$ & 0.1 & NS \\
\hline Crude protein (\%) & 13.6 & 0.1 & 13.9 & 0.1 & $13 \cdot 6$ & 0.1 & $13 \cdot 6$ & 0.1 & $13 \cdot 8$ & 0.1 & NS \\
\hline Ash (\%) & $7.5^{\mathrm{a}}$ & 0.2 & $7.0^{\mathrm{b}}$ & 0.1 & $6 \cdot 8^{\mathrm{b}}$ & 0.1 & $5 \cdot 8^{\mathrm{b}}$ & 0.1 & $8 \cdot 5^{\mathrm{a}}$ & 0.1 & NS \\
\hline Total P (\%) & 0.3 & 0.0 & 0.3 & 0.0 & 0.3 & 0.0 & $0.3^{\mathrm{b}}$ & 0.0 & $0.4^{\mathrm{a}}$ & 0.0 & NS \\
\hline GSH ( $\mu \mathrm{mol} / \mathrm{mg}$ protein) & $7 \cdot 1$ & 0.3 & $7 \cdot 1$ & 0.2 & 8.7 & 0.7 & 7.8 & 0.4 & 7.5 & 0.3 & NS \\
\hline GSSG ( $\mu \mathrm{mol} / \mathrm{mg}$ protein) & $2 \cdot 6$ & 0.2 & $2 \cdot 3$ & 0.0 & $2 \cdot 2$ & 0.1 & $2 \cdot 6^{\mathrm{a}}$ & 0.1 & $2 \cdot 1^{\mathrm{b}}$ & 0.1 & NS \\
\hline GSH:GSSG & $2 \cdot 9^{b}$ & 0.3 & $3 \cdot 1^{\mathrm{b}}$ & 0.1 & $4 \cdot 1^{a}$ & 0.3 & 3.1 & 0.2 & 3.6 & 0.2 & NS \\
\hline \multicolumn{12}{|l|}{ Enzyme activity } \\
\hline Total GPX (mU/mg protein $)^{\star}$ & $19 \cdot 4$ & $1 \cdot 1$ & 19.4 & $1 \cdot 0$ & $18 \cdot 3$ & 1.0 & $17 \cdot 4^{\mathrm{b}}$ & 0.6 & $20 \cdot 7^{a}$ & 1.0 & NS \\
\hline Se-GPX (mU/mg protein) $)^{*}$ & $9 \cdot 3^{\mathrm{b}}$ & 0.7 & $10 \cdot 4^{b}$ & 1.0 & $13 \cdot 2^{\mathrm{a}}$ & 0.5 & $10 \cdot 0$ & 0.6 & 11.9 & 0.6 & $<0.05$ \\
\hline CAT (U/mg protein) $\dagger$ & 41.0 & 1.5 & $41 \cdot 1$ & $2 \cdot 1$ & 39.9 & 1.2 & $37.4^{\mathrm{b}}$ & $1 \cdot 1$ & $43.9^{a}$ & 1.4 & NS \\
\hline \multicolumn{12}{|l|}{ Gene expression } \\
\hline$C A T$ & $0.8^{\mathrm{a}}$ & 0.0 & $0.8^{\mathrm{a}}$ & 0.1 & $0 \cdot 6^{\mathrm{b}}$ & 0.0 & $0.6^{\mathrm{b}}$ & 0.0 & $0.9^{\mathrm{a}}$ & 0.0 & $<0.05$ \\
\hline Gclc & $0.9^{\mathrm{a}}$ & 0.0 & $0.8^{\mathrm{a}}$ & 0.0 & $0.7^{b}$ & 0.0 & 0.8 & 0.0 & 0.8 & 0.0 & $<0.05$ \\
\hline Nrf2 & $1 \cdot 1^{\mathrm{b}}$ & 0.0 & $1 \cdot 2^{\mathrm{a}}$ & 0.0 & $0.9^{c}$ & 0.0 & $1 \cdot 1$ & 0.0 & 1.0 & 0.0 & NS \\
\hline
\end{tabular}

Se0, diets not supplemented with Se; SeS, diets supplemented with sodium selenite; SeY, diets supplemented with Se-enriched yeast; P, plant-based diets; F, fishmeal-based diets; GSH, reduced glutathione; GSSG, oxidised glutathione; GPX, glutathione peroxidase; Se-GPX, Se-dependent glutathione peroxidase; CAT, catalase; Gclc, glutamate-cysteine ligase catalytic subunit; Nrf2, nuclear factor erythroid-2-related factor 2.

${ }_{\text {a,b,c }}$ Mean values within rows and for each diet-related effect (Se supplementation or dietary basis) with unlike superscript letters were significantly different ( $P<0.05$; two-way ANOVA and Student-Newman-Keuls post hoc test). *Equivalent SI unit for $\mathrm{mU} / \mathrm{mg}$ protein for total GPX and Se-GPX is pmol NADPH oxidsed/min per mg protein at $30 \mathrm{C}$.

† Equivalent $\mathrm{SI}$ unit for $\mathrm{U} / \mathrm{mg}$ protein for $\mathrm{CAT}$ is $\mu \mathrm{mol} \mathrm{H}_{2} \mathrm{O}_{2}$ reduced/min per $\mathrm{mg}$ protein 


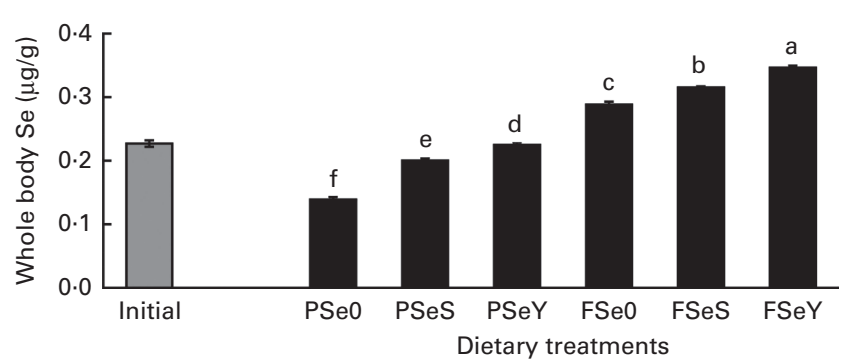

Fig. 1. Total selenium content in initial $(\square)$ and final $(\square)$ whole-body of rainbow trout fry fed the plant-based diet not supplemented with selenium (PSe0), the plant-based diet supplemented with sodium selenite (PSeS), the plant-based diet supplemented with selenium-enriched yeast (PSeY), the fishmeal-based diet not supplemented with selenium (FSe0), the fishmealbased diet supplemented with sodium selenite (FSeS) or the fishmeal-based diet supplemented with selenium-enriched yeast (FSeY) for 12 weeks. Values are means, with their standard errors represented by bars ( $n 3$ tanks per group). ${ }^{\mathrm{a}, \mathrm{b}, \mathrm{c}, \mathrm{d}, \mathrm{e}, \mathrm{f}}$ Mean values with unlike letters were significantly different $(P<0.05$; one-way ANOVA and Student-Newman-Keuls post hoc test).

during the feeding trial. In relation to diet composition, fish fed P-diets displayed higher DM and lipid content and lower ash and P content than fish fed F-diets (Table 3). Higher DM and lipid contents and lower ash content were also noticed in fish fed Se-supplemented diets SeS and SeY, irrespective of dietary basis with plant ingredients (P) or fishmeal (F).
Higher total Se contents were observed in fish fed F-diets and Se-supplemented diets SeS and SeY than in fish fed P-diets and non-supplemented diets Se0, respectively (Fig. 1). Whole-body total Se increased in fish fed Se-enriched yeast (SeY) compared to sodium selenite (SeS), irrespective of the basal diet. Compared to initial first-feeding swim-up fry, the Se concentration was reduced in whole-body of rainbow trout fry fed the plant-based diets PSe0 and PSeS.

\section{Fatty acid composition and lipid peroxidation}

The fatty acid profile of whole rainbow trout fry was significantly modified by dietary basis (Table 4). The changes in fatty acid composition of fry reflected dietary fatty acid composition with lower levels of SFA and $n$-3 PUFA, especially $n$ - 3 long-chain PUFA (18v. 25, $20 v .29$ and $0 v .24 \%$ respectively in P-diets and F-diets) and higher levels of MUFA and $n-6$ PUFA ( $38 v .33$ and 22 v. $4 \%$ respectively) in P-diets, compared to F-diets. The fatty acid profile of whole fish was not significantly affected by dietary Se sources and levels with two exceptions (Table 4). A higher $n-6$ PUFA content (due to a higher $18: 2 n-6$ content) was noticed in fish fed the non-supplemented PSe0 diet compared to other Se-supplemented P-diets PSeS and PSeY, and a lower $n$-3 PUFA content (due to lower $20: 4 n-3,22: 5 n-3$

Table 4. Fatty acid profile (\% total fatty acids) and oxidative status of total lipid from whole rainbow trout fry fed the experimental diets for 12 weeks (Mean values with their standard errors; $n 3$ tanks per diet)

\begin{tabular}{|c|c|c|c|c|c|c|c|c|c|c|c|c|}
\hline & \multicolumn{2}{|c|}{$\mathrm{PSe} 0$} & \multicolumn{2}{|c|}{ PSeS } & \multicolumn{2}{|c|}{ PSeY } & \multicolumn{2}{|c|}{$\mathrm{FSe} 0$} & \multicolumn{2}{|c|}{ FSeS } & \multicolumn{2}{|c|}{ FSeY } \\
\hline & Mean & SEM & Mean & SEM & Mean & SEM & Mean & SEM & Mean & SEM & Mean & SEM \\
\hline \multicolumn{13}{|l|}{ Fatty acid profile } \\
\hline $14: 0$ & $0.4^{\mathrm{c}}$ & 0.0 & $0.4^{\mathrm{c}}$ & 0.0 & $0.4^{\mathrm{c}}$ & 0.0 & $6 \cdot 1^{\mathrm{a}}$ & 0.3 & $5 \cdot 4^{\mathrm{b}}$ & 0.3 & $5 \cdot 3^{b}$ & 0.1 \\
\hline $16: 0$ & $13 \cdot 3^{b}$ & 0.7 & $13 \cdot 3^{b}$ & 0.2 & $13 \cdot 4^{b}$ & 0.6 & $17 \cdot 6^{\mathrm{a}}$ & 0.8 & $16 \cdot 4^{a}$ & 0.5 & $16 \cdot 0^{\mathrm{a}}$ & 0.2 \\
\hline $18: 0$ & $3 \cdot 4^{\mathrm{a}}$ & 0.0 & $3.4^{\mathrm{a}}$ & 0.0 & $3 \cdot 5^{\mathrm{a}}$ & 0.1 & $2 \cdot 9^{\mathrm{b}}$ & 0.0 & $2 \cdot 8^{\mathrm{b}}$ & 0.0 & $2 \cdot 8^{b}$ & 0.1 \\
\hline SFA & $17 \cdot 8^{c}$ & 0.7 & $17 \cdot 9^{c}$ & 0.3 & $18 \cdot 0^{c}$ & 0.6 & $27 \cdot 5^{a}$ & $1 \cdot 0$ & $25 \cdot 5^{\mathrm{a}, \mathrm{b}}$ & 0.7 & $24 \cdot 8^{b}$ & 0.2 \\
\hline $16: 1$ & $0.7^{c}$ & 0.0 & $0.9^{c}$ & 0.0 & $0.9^{c}$ & 0.0 & $8 \cdot 1^{a}$ & 0.4 & $7 \cdot 4^{\mathrm{b}}$ & 0.2 & $7 \cdot 2^{b}$ & 0.1 \\
\hline $18: 1$ & $37 \cdot 1^{a}$ & 0.2 & $37 \cdot 1^{\mathrm{a}}$ & 0.1 & $37 \cdot 0^{a}$ & 0.3 & $17 \cdot 5^{\mathrm{b}}$ & 0.3 & $16 \cdot 9^{b}$ & 0.2 & $16 \cdot 9^{b}$ & 0.3 \\
\hline $20: 1$ & $1 \cdot 1^{b}$ & 0.0 & $1 \cdot 1^{b}$ & 0.0 & $1 \cdot 2^{b}$ & 0.1 & $4 \cdot 5^{a}$ & 0.2 & $4 \cdot 6^{\mathrm{a}}$ & 0.1 & $4 \cdot 7^{\mathrm{a}}$ & 0.1 \\
\hline $22: 1$ & $0 \cdot 1^{c}$ & 0.0 & $0 \cdot 1^{c}$ & 0.0 & $0 \cdot 2^{c}$ & 0.0 & $3 \cdot 8^{b}$ & 0.3 & $4 \cdot 2^{a, b}$ & 0.1 & $4 \cdot 5^{b}$ & 0.1 \\
\hline MUFA & $38.9^{a}$ & 0.2 & $39 \cdot 3^{a}$ & 0.1 & $39 \cdot 3^{a}$ & 0.4 & $33.9^{b}$ & 0.2 & $33 \cdot 2^{b}$ & 0.2 & $33 \cdot 4^{b}$ & 0.3 \\
\hline $18: 2 n-6$ & $19 \cdot 0^{\mathrm{a}}$ & 0.0 & $18 \cdot 7^{b}$ & 0.0 & $18 \cdot 6^{b}$ & 0.1 & $3 \cdot 1^{c}$ & 0.0 & $3 \cdot 1^{\mathrm{c}}$ & 0.0 & $3 \cdot 1^{\mathrm{c}}$ & 0.1 \\
\hline $20: 4 n-6$ & $0.6^{b}$ & 0.0 & $0.6^{b}$ & 0.0 & $0.6^{b}$ & 0.0 & $0.7^{\mathrm{a}}$ & 0.0 & $0.7^{\mathrm{a}}$ & 0.0 & $0.7^{\mathrm{a}}$ & 0.0 \\
\hline n-6 PUFA & $22 \cdot 4^{a}$ & 0.1 & $22 \cdot 1^{b}$ & 0.1 & $22 \cdot 0^{\mathrm{b}}$ & 0.2 & $4 \cdot 5^{c}$ & 0.0 & $4 \cdot 6^{\mathrm{c}}$ & 0.0 & $4 \cdot 6^{c}$ & 0.1 \\
\hline $18: 3 n-3$ & $10 \cdot 8^{a}$ & 0.1 & $10 \cdot 7^{a, b}$ & 0.1 & $10 \cdot 6^{b}$ & 0.0 & $0.9^{c}$ & 0.0 & $0.9^{c}$ & 0.0 & $1 \cdot 0^{c}$ & 0.0 \\
\hline $18: 4 n-3$ & $3 \cdot 5^{\mathrm{a}}$ & 0.1 & $3 \cdot 3^{b}$ & 0.1 & $3 \cdot 2^{b}$ & 0.0 & $1 \cdot 4^{\mathrm{c}}$ & 0.0 & $1.0^{c}$ & 0.0 & $1 \cdot 4^{c}$ & 0.0 \\
\hline $20: 4 n-3$ & $0.4^{\mathrm{c}}$ & 0.0 & $0.5^{c}$ & 0.0 & $0.5^{c}$ & 0.0 & $0.8^{b}$ & 0.0 & $0.9^{a}$ & 0.0 & $0.9^{a}$ & 0.0 \\
\hline $20: 5 n-3$ & $0.8^{b}$ & 0.0 & $0.8^{\mathrm{b}}$ & 0.0 & $0.8^{b}$ & 0.0 & $8 \cdot 0^{a}$ & 0.2 & $8 \cdot 4^{a}$ & 0.1 & $8 \cdot 0^{a}$ & 0.1 \\
\hline $22: 5 n-3$ & $0 \cdot 3^{d}$ & 0.0 & $0.4^{d}$ & 0.0 & $0.4^{d}$ & 0.0 & $0.7^{\mathrm{c}}$ & 0.0 & $2 \cdot 3^{b}$ & 0.0 & $2 \cdot 5^{\mathrm{a}}$ & 0.0 \\
\hline $22: 6 n-3$ & $2 \cdot 8^{\mathrm{C}}$ & 0.2 & $2 \cdot 8^{c}$ & 0.1 & $2 \cdot 8^{c}$ & 0.1 & $11 \cdot 4^{\mathrm{b}}$ & 0.6 & $14 \cdot 5^{\mathrm{a}}$ & 0.4 & $15 \cdot 0^{\mathrm{a}}$ & 0.3 \\
\hline n-3 PUFA & $18 \cdot 9^{c}$ & 0.3 & $18 \cdot 7^{\mathrm{c}}$ & 0.2 & $18 \cdot 5^{\mathrm{c}}$ & $0 \cdot 2$ & $23 \cdot 5^{\mathrm{b}}$ & 0.3 & $28 \cdot 7^{\mathrm{a}}$ & 0.2 & $29 \cdot 3^{a}$ & 0.1 \\
\hline$n$-3 LC-PUFA & $4 \cdot 6^{c}$ & 0.3 & $4 \cdot 7^{\mathrm{C}}$ & 0.2 & $4 \cdot 7^{\mathrm{C}}$ & $0 \cdot 2$ & $21 \cdot 1^{\mathrm{b}}$ & 0.3 & $26 \cdot 3^{a}$ & 0.2 & $27 \cdot 0^{a}$ & 0.2 \\
\hline Unsaturation index & $159^{c}$ & 2 & $158^{c}$ & 1 & $157^{\mathrm{C}}$ & 2 & $176^{\mathrm{b}}$ & 2 & $204^{a}$ & 1 & $208^{a}$ & 1 \\
\hline \multicolumn{13}{|l|}{ Oxidative status } \\
\hline PV & $1 \cdot 8^{\mathrm{b}}$ & 0.4 & $1.9^{b}$ & 0.4 & $3 \cdot 0^{\mathrm{a}}$ & $0 \cdot 2$ & $3 \cdot 2^{\mathrm{a}}$ & 0.1 & $3 \cdot 2^{a}$ & 0.1 & $3 \cdot 2^{a}$ & 0.2 \\
\hline$E_{232}$ & $7 \cdot 0^{\mathrm{b}}$ & 0.2 & $6 \cdot 6^{\mathrm{b}}$ & 0.2 & $6 \cdot 5^{\mathrm{b}}$ & 0.1 & $11 \cdot 2^{\mathrm{a}}$ & 0.2 & $10 \cdot 5^{a}$ & 0.2 & $10 \cdot 7^{a}$ & 0.3 \\
\hline$E_{268}^{208}$ & $2 \cdot 5^{\mathrm{a}, \mathrm{b}}$ & 0.2 & $2 \cdot 2^{\mathrm{a}, \mathrm{b}}$ & 0.1 & $2 \cdot 1^{b}$ & 0.1 & $2 \cdot 7^{\mathrm{a}}$ & 0.2 & $2 \cdot 5^{a, b}$ & 0.1 & $2 \cdot 6^{a, b}$ & 0.2 \\
\hline AV & $2 \cdot 1^{b}$ & 0.0 & $1 \cdot 8^{b}$ & 0.2 & $1 \cdot 5^{\mathrm{b}}$ & 0.2 & $3 \cdot 9^{\mathrm{a}}$ & 0.4 & $3 \cdot 4^{a}$ & 0.4 & $4 \cdot 0^{a}$ & 0.2 \\
\hline LSFP & 9.7 & 0.7 & $9 \cdot 8$ & 0.9 & $9 \cdot 6$ & 0.8 & $11 \cdot 3$ & 0.8 & $10 \cdot 4$ & 0.7 & 11.5 & $1 \cdot 0$ \\
\hline
\end{tabular}

PSe0, plant-based diet not supplemented with Se; PSeS, plant-based diet supplemented with sodium selenite; PSeY, plant-based diet supplemented with Se-enriched yeast: FSe0, fishmeal-based diet not supplemented with Se; FSeS, fishmeal-based diet supplemented with sodium selenite; FSeY, fishmeal-based diet supplemented with Se-enriched yeast; PV, peroxide value expressed as milliequivalents of active oxygen per $\mathrm{kg}$ total lipid; $\mathrm{E}_{232}$, conjugated dienes measured as specific extinction at the wavelength of $232 \mathrm{~nm} ; \mathrm{E}_{268}$, conjugated trienes measured as specific extinction at the wavelength of $268 \mathrm{~nm}$; AV, anisidine value; LSFP, lipid-soluble fluorescent products.

a,b,c,d Mean values within a row with unlike superscript letters were significantly different $(P<0.05$; one-way ANOVA and Student-Newman-Keuls post hoc test). 


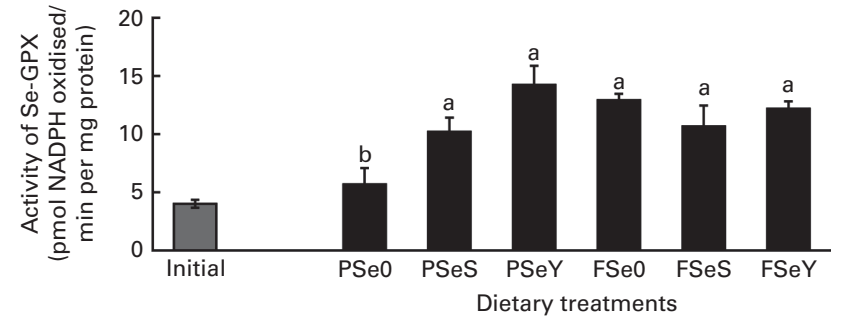

Fig. 2. Activity of selenium-dependent glutathione peroxidase (Se-GPX) in initial $(\square)$ and final $(\square)$ whole-body of rainbow trout fry fed the plant-based diet not supplemented with selenium (PSe0), the plant-based diet supplemented with sodium selenite (PSeS), the plant-based diet supplemented with selenium-enriched yeast (PSeY), the fishmeal-based diet not supplemented with selenium (FSe0), the fishmeal-based diet supplemented with sodium selenite (FSeS) or the fishmeal-based diet supplemented with selenium-enriched yeast (FSeY) for 12 weeks. Values are means, with their standard errors represented by bars ( $n 3$ tanks per group). ${ }^{\mathrm{a}, \mathrm{b}}$ Mean values with unlike letters are significantly different $(P<0.05$; one-way ANOVA and Student-Newman-Keuls post hoc test).

and 22:6n-3 contents) was found in fish fed the nonsupplemented FSe0 diet, compared to other Se-supplemented F-diets FSeS and FSeY.

Some biomarkers of lipid peroxidation such as $\mathrm{E}_{232}$ and anisidine value assessed in total lipid of whole rainbow trout fry were reduced in fish fed P-diets, compared to fish fed F-diets in relation with the oxidative status of diets (16.0 and 2.5 v. 20.5 and 3.0 respectively in P-diets and F-diets). However, the oxidative status of fish was not significantly affected by dietary Se sources and levels (Table 4). On the other hand, GSSG content was significantly higher in fish fed P-diets, compared to fish fed F-diets, and the GSH:GSSG ratio was significantly higher in fish fed SeY-diets, compared to fish fed Se0 or SeS-diets according to two-way ANOVA (Table 3).

\section{Antioxidant enzyme activity and gene expression}

The whole-body Se-GPX activity was significantly lower in rainbow trout fry fed the non-supplemented plant-based diet PSe0 (Fig. 2). According to two-way ANOVA, whole-body Se-GPX activity was significantly higher in fish fed Se-yeast (diets SeY), compared to fish fed sodium selenite (diets SeS) or non-supplemented diets $\mathrm{SeO}$ (Table 3). The activity of other antioxidant enzymes was not significantly affected by dietary Se sources and levels (Table 5). The activity of CAT and total GPX was higher in fish fed F-diets than in fish fed P-diets according to two-way ANOVA (Table 3).

Gene expression profiles were correlated to activity levels of antioxidant enzymes with a significant decreased expression of some Se-GPX (GPX1b1, GPX1b2 and GPX4a1) in rainbow trout fry fed the non-supplemented plant-based diet PSe0, and no effect on the expression of genes coding for the other antioxidant enzymes SOD1, SOD2 and GR (Table 6). Expression of GST was reduced in the PSe0 group only, when compared to the FSe0 group. Expression of SelP was significantly reduced in the PSe0 group, whereas gene expression profiles of the other selenoproteins GPX1a, GPX4a2, GPX4b and TR1 were not affected by dietary Se sources and levels. Expression of the selenoprotein MSRB2 was the lowest in the PSe0 group and the highest in the FSe0 group. On the other hand, according to two-way ANOVA, gene expression of CAT was higher in fish fed F-diets, compared to fish fed P-diets, and gene expressions of $C A T$, glutamate-cysteine ligase catalytic subunit $(G c l c)$ and redox- and electrophile-sensitive transcription factor nuclear factor erythroid-2-related factor 2 ( Nrf2) were higher in fish fed $\mathrm{Se} 0$ and SeS-diets, compared to fish fed SeY-diets (Table 3).

\section{Discussion}

Growth performance of rainbow trout fry was not affected by dietary Se sources and levels ranging from 0.5 to $1.7 \mathrm{mg} / \mathrm{kg}$ diet in accordance with the results reported in juvenile rainbow trout by Hilton et al. ${ }^{(41)}$, who estimated threshold levels at $0.15 \mathrm{mg} / \mathrm{kg}$ diet for requirement and $3 \mathrm{mg} / \mathrm{kg}$ diet for toxicity. Although there was a slight reduction in survival of rainbow trout fry fed fishmeal-based diets with the highest dietary Se content (from 1.2 to $1.7 \mathrm{mg} / \mathrm{kg}$ ), no mortality in relation to dietary Se deficiency was recorded as reported for salmon fry ${ }^{(42)}$. Growth performance was not higher in fish fed F-diets than in fish fed P-diets, as observed in rainbow trout juveniles ${ }^{(43)}$. High dietary Se has been shown to exert toxic effects such as elevated mortality, reduced growth and feed efficiency as well as renal calcinosis at levels marginally

Table 5. Antioxidant enzyme activity of whole rainbow trout fry fed the experimental diets for 12 weeks ${ }^{\star}$

(Mean values with their standard errors; $n 3$ tanks per diet)

\begin{tabular}{|c|c|c|c|c|c|c|c|c|c|c|c|c|}
\hline \multirow[b]{2}{*}{ Antioxidant enzyme } & \multicolumn{2}{|c|}{$\mathrm{PSe} 0$} & \multicolumn{2}{|c|}{ PSeS } & \multicolumn{2}{|c|}{$\mathrm{PSeY}$} & \multicolumn{2}{|c|}{$\mathrm{FSe} 0$} & \multicolumn{2}{|c|}{ FSeS } & \multicolumn{2}{|c|}{$\mathrm{FSeY}$} \\
\hline & Mean & SEM & Mean & SEM & Mean & SEM & Mean & SEM & Mean & SEM & Mean & SEM \\
\hline SOD (U/mg protein) & 4.3 & 0.4 & 4.1 & 0.4 & 3.9 & 0.3 & 4.0 & 0.2 & 4.3 & 0.4 & 4.1 & 0.4 \\
\hline CAT (U/mg protein) $\dagger$ & $35 \cdot 7$ & $2 \cdot 7$ & $40 \cdot 4$ & $2 \cdot 0$ & $36 \cdot 2$ & $1 \cdot 8$ & $46 \cdot 4$ & $2 \cdot 0$ & 41.7 & $4 \cdot 1$ & $43 \cdot 7$ & $2 \cdot 0$ \\
\hline Total GPX (mU/mg protein) & $15 \cdot 6$ & $1 \cdot 1$ & $19 \cdot 0$ & 0.7 & $17 \cdot 5$ & $1 \cdot 7$ & $23 \cdot 2$ & $2 \cdot 1$ & $19 \cdot 8$ & $2 \cdot 1$ & $19 \cdot 0$ & 1.4 \\
\hline $\mathrm{GR}$ (mU/mg protein) & $8 \cdot 2$ & 1.4 & 7.5 & 0.8 & $8 \cdot 7$ & 0.8 & $11 \cdot 1$ & 0.8 & $8 \cdot 6$ & $1 \cdot 2$ & $9 \cdot 2$ & $1 \cdot 2$ \\
\hline GST (mU/mg protein) & $64 \cdot 3$ & $2 \cdot 5$ & $60 \cdot 0$ & $2 \cdot 4$ & $60 \cdot 9$ & 1.5 & $66 \cdot 2$ & $6 \cdot 9$ & $61 \cdot 8$ & 3.9 & 57.9 & 7.9 \\
\hline TR (mU/mg protein) & $1 \cdot 1$ & 0.2 & 0.9 & 0.3 & 1.0 & 0.2 & $1 \cdot 1$ & 0.0 & 0.9 & 0.1 & 1.3 & 0.2 \\
\hline
\end{tabular}

PSe0, plant-based diet not supplemented with Se; PSeS, plant-based diet supplemented with sodium selenite; PSeY, plant-based diet supplemented with Se-enriched yeast;

FSe0, fishmeal-based diet not supplemented with Se; FSeS, fishmeal-based diet supplemented with sodium selenite; FSeY, fishmeal-based diet supplemented with Se-enriched yeast; SOD, superoxide dismutase; CAT, catalase; GPX, glutathione peroxidase; GR, glutathione reductase; GST, glutathione-S-transferase; TR, thioredoxin reductase.

*Mean values within any of the rows were non-significantly different (one-way ANOVA; $P<0.05$ )

† Equivalent $\mathrm{SI}$ unit for $\mathrm{U} / \mathrm{mg}$ protein for $\mathrm{CAT}$ is $\mu \mathrm{mol} \mathrm{H}_{2} \mathrm{O}_{2}$ reduced/min per $\mathrm{mg}$ protein. 
Table 6. Antioxidant enzyme expression of whole rainbow trout fry fed the experimental diets for 12 weeks ${ }^{*}$

(Mean values with their standard errors; $n 3$ tanks per diet)

\begin{tabular}{|c|c|c|c|c|c|c|c|c|c|c|c|c|}
\hline \multirow[b]{2}{*}{ Gene } & \multicolumn{2}{|c|}{$\mathrm{PSe} 0$} & \multicolumn{2}{|c|}{ PSeS } & \multicolumn{2}{|c|}{$\mathrm{PSeY}$} & \multicolumn{2}{|c|}{$\mathrm{FSe} 0$} & \multicolumn{2}{|c|}{ FSeS } & \multicolumn{2}{|c|}{ FSeY } \\
\hline & Mean & SEM & Mean & SEM & Mean & SEM & Mean & SEM & Mean & SEM & Mean & SEM \\
\hline GPX1a & 0.74 & 0.07 & 0.87 & 0.07 & 0.92 & 0.09 & 1.01 & 0.10 & 0.91 & 0.09 & 0.85 & 0.03 \\
\hline$G P X 1 b 1$ & $0.66^{c}$ & 0.05 & $0.87^{a, b, c}$ & 0.07 & $0.75^{\mathrm{b}, \mathrm{c}}$ & 0.04 & $1.00^{\mathrm{a}}$ & 0.07 & $1.09^{a}$ & 0.08 & $0.97^{\mathrm{a}, \mathrm{b}}$ & 0.04 \\
\hline$G P X 1 b 2$ & $0.50^{c}$ & 0.04 & $0.77^{\mathrm{b}}$ & 0.08 & $0.72^{\mathrm{b}}$ & 0.02 & $1.00^{\mathrm{a}}$ & 0.03 & $0.96^{\mathrm{a}}$ & 0.07 & $0.90^{\mathrm{a}, \mathrm{b}}$ & 0.03 \\
\hline$G P X 4 a 1$ & $0.56^{d}$ & 0.04 & $0.89^{a, b}$ & 0.07 & $0.78^{b, c}$ & 0.00 & $1 \cdot 00^{\mathrm{a}}$ & 0.05 & $0.87^{a, b}$ & 0.04 & $0.69^{c, d}$ & 0.03 \\
\hline$G P X 4 a 2$ & 0.82 & 0.04 & 0.84 & 0.09 & 0.86 & 0.08 & 1.01 & 0.09 & 0.85 & 0.10 & 0.91 & 0.05 \\
\hline$G P X 4 b$ & 1.29 & 0.16 & 1.27 & 0.05 & 0.97 & 0.11 & 1.01 & 0.08 & 1.13 & 0.07 & 0.88 & 0.06 \\
\hline SelP & $0.44^{b}$ & 0.01 & $0.86^{a}$ & 0.08 & $0.90^{\mathrm{a}}$ & 0.16 & $1.00^{\mathrm{a}}$ & 0.06 & $0.88^{a}$ & 0.06 & $0.88^{a}$ & 0.05 \\
\hline MSRB2 & $0.60^{c}$ & 0.02 & $0.90^{a, b}$ & 0.06 & $0.82^{a, b, c}$ & 0.08 & $1 \cdot 00^{\mathrm{a}}$ & 0.03 & $0.87^{a, b}$ & 0.09 & $0.64^{b, c}$ & 0.07 \\
\hline TR1 & 0.78 & 0.04 & 0.77 & 0.02 & 0.95 & 0.08 & 1.01 & 0.11 & 0.85 & $0 \cdot 10$ & 0.96 & 0.06 \\
\hline SOD1 & 0.99 & 0.06 & 1.09 & 0.05 & 1.00 & 0.01 & 1.00 & 0.02 & 1.12 & 0.03 & 1.03 & 0.03 \\
\hline SOD2 & 1.46 & 0.13 & 1.72 & 0.11 & 1.30 & 0.04 & 1.00 & 0.07 & 1.34 & 0.18 & $1 \cdot 10$ & 0.06 \\
\hline$C A T$ & $0.56^{b}$ & 0.03 & $0.69^{b}$ & 0.06 & $0.60^{\mathrm{b}}$ & 0.07 & $1.00^{\mathrm{a}}$ & 0.01 & $0.94^{a}$ & 0.10 & $0.69^{b}$ & 0.01 \\
\hline$G R$ & 0.85 & 0.04 & 1.04 & 0.04 & 0.88 & 0.05 & 1.01 & 0.09 & 1.02 & 0.03 & 0.80 & 0.03 \\
\hline GST & $0.89^{b}$ & 0.01 & $1 \cdot 21^{a, b}$ & 0.10 & $1 \cdot 18^{a, b}$ & 0.11 & $1.00^{b}$ & 0.05 & $1 \cdot 39^{a}$ & 0.09 & $1.06^{a, b}$ & 0.02 \\
\hline Gclc & $0.71^{\mathrm{b}}$ & 0.04 & $0.89^{a, b}$ & 0.04 & $0.78^{b}$ & 0.06 & $1.00^{\mathrm{a}}$ & 0.03 & $0.75^{\mathrm{b}}$ & 0.05 & $0.65^{\mathrm{b}}$ & 0.04 \\
\hline Nrf2 & $1 \cdot 10^{\mathrm{a}, \mathrm{b}}$ & 0.05 & $1 \cdot 16^{\mathrm{a}, \mathrm{b}}$ & 0.07 & $0.91^{\mathrm{b}}$ & 0.05 & $1.00^{\mathrm{a}, \mathrm{b}}$ & 0.06 & $1 \cdot 21^{\mathrm{a}}$ & 0.06 & $0.93^{b}$ & 0.05 \\
\hline
\end{tabular}

PSe0, plant-based diet not supplemented with Se; PSeS, plant-based diet supplemented with sodium selenite; PSeY, plant-based diet supplemented with Se-enriched yeast FSe0, fishmeal-based diet not supplemented with Se; FSeS, fishmeal-based diet supplemented with sodium selenite; FSeY, fishmeal-based diet supplemented with Se-enriched yeast; GPX, glutathione peroxidase; SeIP, selenoprotein P; MSRB, methionine-R-sulphoxide reductase B; TR, thioredoxin reductase; SOD, superoxide dismutase; CAT, catalase; GR, glutathione reductase; GST, glutathione-S-transferase, Gclc, glutamate-cysteine ligase catalytic subunit; Nrf2, nuclear factor erythroid-2-related factor 2.

a,b,c,d Mean values within a row with unlike superscript letters are significantly different $(P<0.05$; one-way ANOVA and Student-Newman-Keuls post hoc test). * Transcript expression was normalised to $\beta$-actin RNA and values are expressed as a ratio of the FSe0 group.

above those required ${ }^{(13)}$, but the low survival of fish fed F-diets is probably more indicative of another dietary mineral deficiency. Indeed, the F-diets were not supplemented with mineral mixture, whereas the P-diets contained $1 \%$ mineral mixture. Fish meal-based diets contained only $10 \mathrm{mg} \mathrm{Mn} / \mathrm{kg}$ diet, slightly lower than the estimated requirement level of $12-13 \mathrm{mg} / \mathrm{kg}^{(13)}$, whereas the plant-based diets contained $80 \mathrm{mg} / \mathrm{kg}^{(43)}$. A fishmeal-based diet without Mn supplement has been shown to cause poor hatchability in rainbow trout broodstock $^{(44)}$.

Uptake of Se by fish can be from water or diet ${ }^{(16,45)}$. The waterborne Se content of $2 \cdot 2 \mu \mathrm{g} / 1$ determined at the beginning of the present study according to Darrouzès et al. ${ }^{(46,47)}$ was within the range that rarely produced discernible adverse effects on fish according to Hamilton ${ }^{(45)}$. This level was also unlikely to contribute significantly to the dietary requirement for Se even if waterborne Se has been shown to be readily accumulated by rainbow trout ${ }^{(48)}$. The lowest whole-body Se content was found in fish fed the non-supplemented plantbased diet PSe0. The whole-body Se concentration of these fish was particularly low, as it was lower compared to initial fish from the present study or rainbow trout juveniles fed a similar diet ${ }^{(43)}$. A reduced tissue Se concentration associated with a low GPX activity has been reported in rainbow trout fed Se-deficient diet ${ }^{(17,41,49-51)}$ and in other fish species ${ }^{(52-58)}$.

However, the total Se accumulation in fish tissues (except for kidney) has been shown to be dose-dependent ${ }^{(59)}$, and thus a reduced whole-body Se content is indicative of a reduced dietary Se level, but not necessarily of a Se-deficient diet.

A dietary Se deficiency has generally been reported to result in growth reduction as well as reduced activity of $\mathrm{GPX}^{(13)}$. Hepatic GPX activity has been found to be a more stringent and more robust criterion than weight gain to define Se deficiency ${ }^{(60)}$. In the present study, due to the small size of fish, Se-GPX activity was determined in the whole-body of rainbow trout fry and not in liver. A lower whole-body Se-GPX activity was noticed in rainbow trout fry fed the non-supplemented plant-based diet $\mathrm{PSe} 0$, suggesting a dietary Se deficiency in this group of rainbow trout fry fed $0.5 \mathrm{mg}$ $\mathrm{Se} / \mathrm{kg}$ diet. This result also suggests the possibility of using whole-body Se-GPX activity to define Se deficiency in small fish. Based on plasma GPX activity and using purified diets supplemented with sodium selenite, Hilton et al. ${ }^{(41)}$ defined the Se requirement at $0.38 \mathrm{mg} / \mathrm{kg}$ for rainbow trout juveniles. The Se requirement for fish is known to be affected by the type of Se source ${ }^{(60)}$. Organic sources such as selenomethionine or Se-enriched yeast but not selenocysteine have been demonstrated to be more available than inorganic sources such as sodium selenite ${ }^{(60)}$. Se availability from plant-derived proteins is reported to be considerably higher than that from fishmeals ${ }^{(16)}$. Wheat and soyabean used to formulate P-diets are supposed to contain predominantly selenomethionine with lesser amounts of selenocysteine and selenate ${ }^{(23)}$. So the higher Se requirement of fish from the present study compared to the study of Hilton et al. ${ }^{(41)}$ might not be attributed to a lower Se availability from the PSe0 diet compared to sodium selenite. The Se requirement for fish is also known to be affected by interaction between dietary vitamin $\mathrm{E}$ and $\mathrm{Se}^{(13,60)}$. However, the dietary vitamin $\mathrm{E}$ requirement of rainbow trout was assumed to be fulfilled in the present study with the supplementation of $50 \mathrm{mg} \alpha$-tocopherol acetate $/ \mathrm{kg}$ diet $^{(13)}$. On the other hand, Bell et al. ${ }^{(17)}$ suggested a higher dietary Se requirement in early life stages to explain the absence of pathology in rainbow trout juveniles fed low dietary Se levels in their study compared to the only available study carried out with early developmental stages of fish by 
Poston et $a l^{(42)}$. The early developmental stages that are characterised by a high growth rate are also more susceptible to oxidative stress than the juvenile stage, possibly due to delayed response or lack of complete development of endogenous antioxidant defence system, and so rainbow trout may need more dietary antioxidant micronutrients at the beginning of their life ${ }^{(31,37)}$. Recent studies have shown beneficial effects of high dietary Se supplementation (between 3 and $5 \mathrm{mg} \mathrm{Se} / \mathrm{kg}$ ) for fish larvae ${ }^{(55,61,62)}$, whereas the tested levels were similar to the threshold levels defined for adverse effects in fish juveniles ${ }^{(13)}$, suggesting differences in Se metabolism of fish larvae and of juveniles.

The reduction of whole-body Se-GPX activity in the PSe0 group did not lead to an increased compensatory wholebody GST activity contrary to findings in liver and plasma of juvenile rainbow trout or channel catfish ${ }^{(15,49,50)}$. However, as total GPX was not significantly different between groups, an induction of the non-Se-dependent GPX can be assumed. The reduced activity of Se-GPX in fish fed PSe0 was associated with concomitant decreased expression of the Se-GPX gene isoforms GPX1b1, GPX1b2 and GPX4a1 confirming the observations on GPX transcript expression in rainbow trout hepatocytes $^{(6)}$, zebrafish ${ }^{(58)}$, cod larvae ${ }^{(61)}$ and rats ${ }^{(63)}$. In contrast, GPX1a did not show any significant difference in expression level contrary to what has been reported in vitro with GPX1a being more induced by Se at low/intermediate concentrations, and GPX1b1 and GPX1b2 at high concentrations ${ }^{(6)}$. GPX4 appeared to be less sensitive to Se exposure with GPX4a1 being the most sensitive isoform in rainbow trout hepatocytes ${ }^{(6)}$, whereas the lack of regulation of GPX4 by Se has been reported in cod larvae $e^{(61)}$ and mammals ${ }^{(3,63,64)}$.

Other selenoproteins such as TR were not affected by dietary Se sources and levels, except for SelP whose expression was lower in fish fed the PSe0 diet. These results seem to be in contradiction to the two studies in rainbow trout juveniles fed fishmeal-based diets where a higher hepatic TR activity was noticed in fish fed diets supplemented with the highest level of Se-enriched yeast ${ }^{(19,65)}$. This is possibly due to the fact that the dietary Se levels tested were different between the three studies: from 0.5 to $1.7 \mathrm{mg} / \mathrm{kg}$ diet in the present study as against $0.7-7 \cdot 4$ in the studies of Rider et al. ${ }^{(19,65)}$. As the Se levels tested in the latter studies were above requirements, GPX activity was not affected ${ }^{(19,65)}$. The dietary Se levels tested in the present study might not be high enough to observe an impact on whole-body TR activity and gene expression. A hierarchy of selenoprotein expression has been recognised in mammals when Se is limiting with a preferential expression of essential selenoproteins such as TR1 compared to stress-related selenoproteins such as GPX1 and SelP ${ }^{(12,66)}$ in accordance with the results of the present study.

The genes of some antioxidant enzymes (GST, glutamatecysteine ligase, SOD1) and the selenoprotein TR1 contain the antioxidant response element in their promoter regions, and can thus be induced by activation of the transcription factor $\mathrm{Nrf} 2^{(67)}$. Different mechanisms appear to be involved in Nrf2 activation, including both post-transcriptional and transcriptional events, as an increase in $N r f 2$ gene transcription has been reported in certain types of cells or physiological conditions $^{(67)}$. In the present study, GST, SOD1 and TR1 mRNA levels were not affected by dietary Se sources and levels in contrast to the results of Burk et al. ${ }^{(68)}$ who reported an induction of GST by dietary Se deficiency through Nrf2-antioxidant response element in mouse liver. We found that $N r f 2$ was induced in fish fed the non-supplemented diets Se0, compared to fish fed Se-enriched yeast, but was repressed compared to fish fed sodium selenite. Only glutamate-cysteine ligase was induced in fish fed the FSe0-diet, compared to fish fed FSeS or FSeY-diets. This discrepancy could be related to a difference in dietary Se levels in mouse fed a Se-deficient diet containing less than $0.01 \mathrm{mg}$ $\mathrm{Se} / \mathrm{kg} v .0 .5 \mathrm{mg} \mathrm{Se} / \mathrm{kg}$ in the present study, which was possibly not deficient enough to observe an impact on the Nrf2-antioxidant response element pathway. However, it could also be related to a difference in the level of induced oxidative stress in animals fed non supplemented Se-diets, since we observe a higher $\mathrm{Nrf2}$ and Gclc gene expression in trout fed the FSe0 diet, compared to those fed the FSeY diet. Fish fed F-diets were characterised by a higher lipid peroxidation level than fish fed P-diets, which might be necessary to promote the activation of $\mathrm{Nrf} 2$.

The lipid peroxidation status was higher in lipids of rainbow trout fry fed F-diets, but was not significantly affected by dietary Se sources and levels. These results are consistent with previous observations in fingerling channel catfish ${ }^{(15)}$ and common carp fed 0 or $1.5 \mathrm{mg} / \mathrm{kg}$ of diphenyl diselenide ${ }^{(69)}$. However, dietary Se supplementation has been shown to reduce lipid peroxidation in juvenile rainbow trout $^{(17,51)}$, sea bass larvae ${ }^{(62)}$ and common carp fed 0 or $3 \mathrm{mg} / \mathrm{kg}$ of diphenyl diselenide ${ }^{(69)}$. Differences could be due to the tested dietary Se levels, since the antioxidant effect of Se was seen in common carp at $3 \mathrm{mg} / \mathrm{kg}$ but not at $1.5 \mathrm{mg} / \mathrm{kg}^{(69)}$, and Se has also been shown to be a prooxidant at high levels in several fish species ${ }^{(65)}$ such as $5 \mathrm{mg} / \mathrm{kg}$ for common carp ${ }^{(69)}$. Differences could also be due to the form of Se and the sensitivity of the marker used to assess lipid peroxidation, since the GSH:GSSG ratio was higher in rainbow trout fry fed Se-yeast than in fish fed sodium selenite or nonsupplemented diets, contrary to the other markers that were not significantly affected by dietary Se sources and levels. The lack of effect of supplemental Se on these markers of lipid peroxidation may also indicate that the decrease of the antioxidant status noticed in fish fed the non-supplemented plant-based diet $\mathrm{PSe} 0$ is not critical enough to provoke oxidative stress in the low stressful conditions used in the present study (e.g. low stocking density), as also reported by the observations of Küçükbay et al. ${ }^{(51)}$.

Present results highlight the influence of the dietary Se form on whole-body Se content and activity of GPX in rainbow trout fry, confirming data on the greater bioavailability of organic Se compared to inorganic sources ${ }^{(18,19,51,60)}$. Moreover, a higher GSH:GSSG ratio and a decreased CAT, glutamate-cysteine ligase and Nrf2 gene expression indicative of decreased oxidative stress was noticed in rainbow trout fry fed Se-yeast compared to sodium selenite, confirming the antioxidant properties of some selenoproteins that were affected by the organic Se levels tested in the present study. 
In conclusion, the present work indicates that feeding rainbow trout fry with plant-based diets without Se supplementation might be detrimental to the antioxidant status of fish. It also confirms the superiority of organic forms to fulfil the dietary Se requirement of rainbow trout fry. It also supports the observations that whole-body GPX and SelP expression are good markers of Se deficiency in rainbow trout fry.

\section{Acknowledgements}

The authors wish to thank F. Terrier, F. Sandres and Y. Hontang for the preparation of diets and care of fish. They are also very grateful to V. Véron, L. Larroquet, M. Cluzeaud, A. Surget and M.-J. Borthaire for technical assistance.

The present study was supported by the INRA-WUR aquaculture platform (P. A. J. P., PhD fellowship), China Scholarship Council (H. L., grant no. 201204910354) and Conseil Régional d'Aquitaine (S. G., B. B. and M. B., grant no. 20071303002PFM).

The authors' contributions are as follows: S. F.-D., P. A. J. P., P. T., F. M. and S. J. K. designed the study; S. G. and H. L. conducted the study; S. F.-D., B. B. and M. B. analysed the data; S. F.-D., S. G. and S. J. K. wrote the article; S. F.-D. had primary responsibility for the final content. All authors read and approved the final manuscript. The authors declare that there are no conflicts of interest.

\section{References}

1. Rayman MP (2000) The importance of selenium to human health. Lancet 356, 233-241.

2. Papp LV, Lu J, Holmgren A, et al. (2007) From selenium to selenoproteins: synthesis, identity, and their role in human health. Antioxid Redox Signal 9, 775-806.

3. Brigelius-Flohé R (1999) Tissue-specific functions of individual glutathione peroxidases. Free Radic Biol Med 27, 951-965.

4. Lobanov AV, Hatfield DL \& Gladyshev VN (2008) Reduced reliance on the trace element selenium during evolution of mammals. Genome Biol 9, R62.

5. Arthur JR (2000) The glutathione peroxidases. Cell Mol Life Sci 57, 1825-1835.

6. Pacitti D, Wang T, Page MM, et al. (2013) Characterization of cytosolic glutathione peroxidase and phospholipidhydroperoxide glutathione peroxidase genes in rainbow trout (Oncorbynchus mykiss) and their modulation by in vitro selenium exposure. Aquat Toxicol 130-131, 97-111.

7. Crosley LK, Méplan C, Nicol F, et al. (2007) Differential regulation of expression of cytosolic and mitochondrial thioredoxin reductase in rat liver and kidney. Arch Biochem Biophys 459, 178-188.

8. Kim H-Y (2013) The methionine sulfoxide reduction system: selenium utilization and methionine sulfoxide reductase enzymes and their functions. Antioxid Redox Signal 19, 958-969.

9. Takebe G, Yarimizu J, Saito Y, et al. (2002) A comparative study on the hydroperoxide and thiol specificity of the glutathione peroxidase family and selenoprotein P. J Biol Chem 277, 41254-41258.

10. Steinbrenner H, Bilgic E, Alili L, et al. (2006) Selenoprotein P protects endothelial cells from oxidative damage by stimulation of glutathione peroxidase expression and activity. Free Radic Res 40, 936-943.

11. Hesketh J (2008) Nutrigenomics and selenium: gene expression patterns, physiological targets, and genetics. Ann Rev Nutr 28, 157-177.

12. Mariotti M, Ridge PG, Zhang Y, et al. (2012) Composition and evolution of the vertebrate and mammalian selenoproteomes. PLOS ONE 7, e33066.

13. Committee on the Nutrient Requirements of Fish and Shrimp \& National Research Council (2011) Nutrient Requirements of Fish and Shrimp. Washington, DC: National Academies Press.

14. Wang KY, Peng CZ, Huang JL, et al. (2013) The pathology of selenium deficiency in Cyprinus carpio L. J Fish Dis 36, 609-615.

15. Gatlin DM, Poe WE \& Wilson RP (1986) Effects of singular and combined dietary deficiencies of selenium and vitamin $\mathrm{E}$ on fingerling channel catfish (Ictalurus punctatus). J Nutr 116, 1061-1067.

16. Watanabe T, Kiron V \& Satoh S (1997) Trace minerals in fish nutrition. Aquaculture 151, 185-207.

17. Bell JG, Cowey CB, Adron JW, et al. (1985) Some effects of vitamin $\mathrm{E}$ and selenium deprivation on tissue enzyme levels and indices of tissue peroxidation in rainbow trout (Salmo gairdneri). Br J Nutr 53, 149-157.

18. Jaramillo FJ, Peng L \& Gatlin DM III (2009) Selenium nutrition of hybrid striped bass (Morone chrysops $\times$ M. saxatilis) bioavailability, toxicity and interaction with vitamin E. Aquac Nutr 15, 160-165.

19. Rider SA, Davies SJ, Jha AN, et al. (2010) Bioavailability of co-supplemented organic and inorganic zinc and selenium sources in a white fishmeal-based rainbow trout (Oncorbynchus mykiss) diet. J Anim Physiol Anim Nutr 94, 99-110.

20. US Food and Drug Administration (2014) Food Additives Permitted in Feed and Drinking Water of Animals-Selenium. Code of Federal Regulations, Part 573, Sec. 573.920

21. European Food Safety Authority - Panel on Additives and Products or Substances Used in Animal Feed (2014) Scientific opinion on the safety and efficacy of DL-selenomethionine as a feed additive for all animal species. EFSA J 12, 3567.

22. Tacon AGJ \& Metian M (2008) Global overview on the use of fish meal and fish oil in industrially compounded aquafeeds: trends and future prospects. Aquaculture 285, 146-158.

23. Rayman MP, Infante HG \& Sargent M (2008) Food-chain selenium and human health: spotlight on speciation. $\mathrm{Br} \mathrm{J}$ Nutr 100, 238-253.

24. Naganuma A, Tanaka T, Maeda K, et al. (1983) The interaction of selenium with various metals in vitro and in vivo. Toxicology 29, 77-86.

25. Feroci G, Fini A, Badiello R, et al. (1997) Interaction between selenium derivatives and heavy metal ions: $\mathrm{Cu}^{2+}$ and $\mathrm{Pb}^{2+}$. Microchem J 57, 379-388.

26. Feroci G, Badiello R \& Fini A (2005) Interactions between different selenium compounds and zinc, cadmium and mercury. J Trace Elem Med Biol 18, 227-234.

27. European Committee for Standardization (1994) Water Quality - Determination of Kjeldabl Nitrogen - Method after Mineralization with Selenium. Brussels: CEN (ISO 5663:1984).

28. Folch J, Lees M \& Sloane Stanley GH (1957) A simple method for the isolation and purification of total lipides from animal tissues. J Biol Chem 226, 497-509.

29. AFNOR (1992) Viandes, préparations de viande et produits à base de viande - Détermination de la teneur en phosphore total. (Meats, Meat Preparations and Meat-based 
Products-Determination of Total Phosphorus Level). La Plaine Saint-Denis: AFNOR Editions (NF V04-406).

30. Pedrero Z, Murillo S, Camara C, et al. (2011) Selenium speciation in different organs of African catfish (Clarias gariepinus) enriched through a selenium-enriched garlic based diet. J Anal At Spectrom 26, 116-125.

31. Fontagné S, Lataillade E, Brèque J, et al. (2008) Lipid peroxidative stress and antioxidant defence status during ontogeny of rainbow trout (Oncorbynchus mykiss). Br J Nutr 100, 102-111.

32. Shantha NC \& Decker EA (1994) Rapid, sensitive, iron-based spectrophotometric methods for determination of peroxide values of food lipids. $J A O A C$ Int 77, 421-424.

33. European Committee for Standardization (2002) Animal and Vegetable Fats and Oils - Determination of Ultraviolet Absorbance Expressed as Specific UV Extinction. Brussels: CEN (ISO 3656:2002).

34. European Committee for Standardization (2000) Animal and Vegetable Fats and Oils - Determination of Anisidine Value. Brussels: CEN (ISO 6885:2000).

35. Hammer C \& Braum E (1988) Quantification of age pigments (lipofuscin). Comp Biochem Physiol B Biochem Mol Biol 90, 7-17.

36. Mourente G \& Diaz-Salvago E (1998) Lipid composition and oxidation status in brain of wild-caught size-class distributed Parapenaeus longirostris (Lucas, 1846). Comp Biochem Physiol B Biochem Mol Biol 120, 457-466.

37. Fontagné-Dicharry S, Lataillade E, Surget A, et al. (2014) Antioxidant defense system is altered by dietary oxidized lipid in first-feeding rainbow trout (Oncorbynchus mykiss). Aquaculture 424-425, 220-227.

38. Lowry OH, Rosebrough NJ, Farr AL, et al. (1951) Protein measurement with the Folin-phenol reagent. $J$ Biol Chem 193, 265-275.

39. Daprà F, Geurden I, Corraze G, et al. (2011) Physiological and molecular responses to dietary phospholipids vary between fry and early juvenile stages of rainbow trout (Oncorbynchus mykiss). Aquaculture 319, 377-384.

40. Pfaffl MW (2001) A new mathematical model for relative quantification in real-time RT-PCR. Nucleic Acids Res 29, e45.

41. Hilton JW, Hodson PV \& Slinger SJ (1980) The requirement and toxicity of selenium in rainbow trout (Salmo gairdneri). J Nutr 110, 2527-2535.

42. Poston HA, Combs GF \& Leibovitz L (1976) Vitamin E and selenium interrelations in the diet of Atlantic salmon (Salmo salar): gross, histological and biochemical deficiency signs. J Nutr 106, 892-904.

43. Antony Jesu Prabhu P, Schrama JW, Mariojouls C, et al. (2014) Post-prandial changes in plasma mineral levels in rainbow trout fed a complete plant ingredient based diet and the effect of supplemental di-calcium phosphate. Aquaculture 430, 34-43.

44. Takeuchi T, Watanabe T, Ogino C, et al. (1981) Effects of low protein-high calorie diets and deletion of trace elements from a fish meal diet on reproduction of rainbow trout. Bull Jpn Soc Sci Fish 47, 645-654.

45. Hamilton SJ (2004) Review of selenium toxicity in the aquatic food chain. Sci Total Environ 326, 1-31.

46. Darrouzès J, Bueno M, Simon S, et al. (2008) Advantage of hydride generation interface for selenium speciation in waters by high performance liquid chromatography - inductively coupled plasma mass spectrometry coupling. Talanta 75, 362-368.

47. Darrouzès J, Bueno M, Lespes G, et al. (2005) Operational optimisation of ICP-octopole collision/reaction cell-MS for applications to ultratrace selenium total and speciation determination. J Anal At Spectrom 20, 88-94.

48. Hodson PV, Hilton JW \& Slinger SJ (1986) Accumulation of waterborne selenium by rainbow trout (Salmo gairdneri), eggs, fry and juveniles. Fish Physiol Biochem 1, 187-196.

49. Bell JG, Pirie BJ, Adron JW, et al. (1986) Some effects of selenium deficiency on glutathione peroxidase (EC 1.11.1.9) activity and tissue pathology in rainbow trout (Salmo gairdneri). Br J Nutr 55, 305-311.

50. Bell JG, Adron JW \& Cowey CB (1986) Effect of selenium deficiency on hydroperoxide-stimulated release of glutathione from isolated perfused liver of rainbow trout (Salmo gairdneri). Br J Nutr 56, 421-428.

51. Küçükbay FZ, Yazlak H, Karaca I, et al. (2009) The effects of dietary organic or inorganic selenium in rainbow trout (Oncorbynchus mykiss) under crowding conditions. Aquac Nutr 15, 569-576.

52. Wang Y, Han J, Li W, et al. (2007) Effect of different selenium source on growth performances, glutathione peroxidase activities, muscle composition and selenium concentration of allogynogenetic crucian carp (Carassius auratus gibelio). Anim Feed Sci Technol 134, 243-251.

53. Zhou XX, Wang YB, Gu Q, et al. (2009) Effects of different dietary selenium sources (selenium nanoparticle and selenomethionine) on growth performance, muscle composition and glutathione peroxidase enzyme activity of crucian carp (Carassius auratus gibelio). Aquaculture 291, $78-81$.

54. Han D, Xie S, Liu M, et al. (2011) The effects of dietary selenium on growth performances, oxidative stress and tissue selenium concentration of gibel carp (Carassius auratus gibelio). Aquac Nutr 17, e741-e749.

55. Ribeiro ARA, Ribeiro L, Sxle $\varnothing$, et al. (2012) Selenium supplementation changes glutathione peroxidase activity and thyroid hormone production in Senegalese sole (Solea senegalensis) larvae. Aquac Nutr 18, 559-567.

56. Zhu Y, Chen Y, Liu Y, et al. (2012) Effect of dietary selenium level on growth performance, body composition and hepatic glutathione peroxidase activities of largemouth bass Micropterus salmoide. Aquac Res 43, 1660-1668.

57. Le KT, Fotedar R \& Partridge G (2014) Selenium and vitamin $\mathrm{E}$ interaction in the nutrition of yellowtail kingfish (Seriola lalandi): physiological and immune responses. Aquac Nutr 20, 303-313.

58. Penglase S, Hamre K, Rasinger JD, et al. (2014) Selenium status affects selenoprotein expression, reproduction, and F-1 generation locomotor activity in zebrafish (Danio rerio). Br J Nutr 111, 1918-1931.

59. Lee S, Lee J-H, Bai SC, et al. (2010) Evaluation of the dietary toxic level of selenium (Se) in juvenile olive flounder, Paralichthys olivaceus. J World Aquacult Soc 41, 245-254.

60. Antony Jesu Prabhu P, Schrama JW \& Kaushik SJ (2014) Mineral requirements of fish: a systematic review. Rev Aquac (epublication 5 December 2014).

61. Penglase S, Nordgreen A, van der Meeren T, et al. (2010) Increasing the level of selenium in rotifers (Brachionus plicatilis 'Cayman') enhances the mRNA expression and activity of glutathione peroxidase in cod (Gadus morbua L.) larvae. Aquaculture 306, 259-269.

62. Betancor MB, Caballero M, Terova G, et al. (2012) Selenium inclusion decreases oxidative stress indicators and muscle injuries in sea bass larvae fed high-DHA microdiets. $\mathrm{Br} \mathrm{J}$ Nutr 108, 2115-2128.

63. Barnes KM, Evenson JK, Raines AM, et al. (2009) Transcript analysis of the selenoproteome indicates that dietary 
selenium requirements of rats based on selenium-regulated selenoprotein mRNA levels are uniformly less than those based on glutathione peroxidase activity. J Nutr 139, 199-206.

64. Zhou J-C, Zhao H, Li J-G, et al. (2009) Selenoprotein gene expression in thyroid and pituitary of young pigs is not affected by dietary selenium deficiency or excess. $J$ Nutr 139, 1061-1066.

65. Rider SA, Davies SJ, Jha AN, et al. (2009) Supra-nutritional dietary intake of selenite and selenium yeast in normal and stressed rainbow trout (Oncorbynchus mykiss): implications on selenium status and health responses. Aquaculture 295 , $282-291$.
66. Schomburg L \& Schweizer U (2009) Hierarchical regulation of selenoprotein expression and sex-specific effects of selenium. Biochim Biophys Acta 1790, 1453-1462.

67. Kwak MK, Wakabayashi N \& Kensler TW (2004) Chemoprevention through the Keap1-Nrf2 signaling pathway by phase 2 enzyme inducers. Mutat Res 555, 133-148.

68. Burk RF, Hill KE, Nakayama A, et al. (2008) Selenium deficiency activates mouse liver Nrf2-ARE but vitamin E deficiency does not. Free Radic Biol Med 44, 1617-1623.

69. Menezes C, Leitemperger J, Santi A, et al. (2014) Evaluation of the effects induced by dietary diphenyl diselenide on common carp Cyprinus carpio. Fish Physiol Biochem 40, $141-149$. 